\title{
Postoperative visits by dedicated anesthesiologists in patients with elevated troponin: a retrospective cohort study evaluating postoperative care utility and early detection of complications
}

\author{
Annemarie Akkermans ${ }^{1 \dagger}$, Lisette M. Vernooij ${ }^{1},^{2 *+}$ (D), Wilton A. van Klei ${ }^{1}$ and Judith A. van Waes ${ }^{1}$
}

\begin{abstract}
Background: An elevated cardiac troponin level after noncardiac surgery is associated with both morbidity and mortality. Guidelines suggest routine troponin monitoring in high-risk patients. We implemented a dedicated anesthesia team to conduct follow-up on patients with postoperative troponin elevation. We hypothesized that these visits would facilitate early detection of complications. Therefore, the aim of this study was to evaluate the effect of postoperative visits by dedicated anesthesiologists on early detection of complications and care utility.

Methods: This retrospective observational study included patients aged $\geq 60$ years with an elevated troponin within the first 3 days after noncardiac surgery. Troponin elevation was detected by routine biomarker monitoring. The primary outcome was early detected myocardial infarctions by the dedicated anesthesiologist. Other outcomes were overall detected complications, additional diagnostic tests and treatment advised by the anesthesiologist, consultation of another medical specialist, and advised postoperative follow-up at the outpatient cardiac clinic within 1 week after surgery.

Results: Of the 811 patients, 509 (63\%) received a postoperative consultation by the anesthesiologist. Anesthesiologists were involved in the early detection of $59 \%$ of all myocardial infarctions and in $12 \%$ of all complications. Besides cardiac ischemia, patients were also often diagnosed with noncardiac complications, including respiratory failure (8.9\%), pneumonia (13.2\%), and acute kidney injury (17.5\%) within 1 week after surgery. In $75 \%$ of patients, anesthesiologists ordered additional diagnostics, most frequently existing of electrocardiograms and additional cardiac enzyme testing. Additionally, change in treatment was advised, most often a medication change, in $16 \%$ of patients.

(Continued on next page)
\end{abstract}

\footnotetext{
* Correspondence: I.m.vernooij@umcutrecht.nl

${ }^{\dagger}$ Annemarie Akkermans and Lisette M. Vernooij contributed equally to this work.

'Department of Anesthesiology, University Medical Centre Utrecht, Utrecht University, Utrecht, the Netherlands

${ }^{2}$ Department of Epidemiology, Julius Centre for Health Sciences and Primary Care, University Medical Centre Utrecht, Utrecht University, Utrecht, The Netherlands
}

C C The Author(s). 2020 Open Access This article is licensed under a Creative Commons Attribution 4.0 International License, which permits use, sharing, adaptation, distribution and reproduction in any medium or format, as long as you give appropriate credit to the original author(s) and the source, provide a link to the Creative Commons licence, and indicate if changes were made. The images or other third party material in this article are included in the article's Creative Commons licence, unless indicated otherwise in a credit line to the material. If material is not included in the article's Creative Commons licence and your intended use is not permitted by statutory regulation or exceeds the permitted use, you will need to obtain permission directly from the copyright holder. To view a copy of this licence, visit http://creativecommons.org/licenses/by/4.0/ The Creative Commons Public Domain Dedication waiver (http://creativecommons.org/publicdomain/zero/1.0/) applies to the data made available in this article, unless otherwise stated in a credit line to the data. 
(Continued from previous page)

Conclusions: Standard consultation of a dedicated anesthesiologist resulted in an early detection of 59\% of all myocardial infarctions and involved a change in treatment in a considerable number of patients with postoperative troponin elevation. Whether this may improve patient outcomes remains to be elucidated.

Keywords: Troponin, Perioperative anesthesiologist, Screening, Myocardial infarction

\section{Introduction}

An increased serum cardiac troponin level, as a marker for postoperative myocardial injury, has been shown to be an independent predictor of morbidity and mortality within the first year after non-cardiac surgery (Devereaux et al. 2017; Levy et al. 2011; van Waes et al. 2016; van Waes et al. 2013). Depending on the amount of troponin elevation and the surgical population, the incidence of mortality within 30 days varies between 4 and 17\% (Devereaux et al. 2017; Devereaux et al. 2012; van Waes et al. 2013). As only $15 \%$ of patients with postoperative myocardial injury experiences typical ischemic symptoms (Devereaux et al. 2011), routine postoperative troponin surveillance is recommended by several guidelines for postoperative risk stratification to direct secondary prevention (Duceppe et al. 2017; Thygesen et al. 2018).

In our center, we implemented a routine postoperative troponin I (TnI) surveillance program in January 2011. In a previous study in which this program was evaluated, it became apparent that only $41 \%$ of all patients with postoperative myocardial injury received cardiac consultation (van Waes et al. 2016). Cardiac etiology was suspected in almost half of the consulted patients. However, troponin elevation has also been related to other disease entities including stroke, sepsis, and pulmonary embolism (Akkermans et al. 2019; Beattie et al. 2018; Noordzij et al. 2015; van Waes et al. 2016). We hypothesized that a more general approach to assess postoperative troponin elevation, rather than an approach focusing on cardiac etiology, would be beneficial. As a result, in October 2016, we implemented routine postoperative consultations by a dedicated anesthesia team in patients detected with an elevated troponin in the surveillance program. The goal of these visits was to improve postoperative follow-up and to potentially detect or prevent complications at an early stage. We hypothesized that such routine visits would facilitate early detection of complications with only a limited use of resources. Therefore, this study aimed to evaluate the effect of these visits by dedicated anesthesiologists on early detection of complications and postoperative care utility in patients with troponin elevation after noncardiac surgery.

\section{Methods}

This study was conducted in adherence to the STROBE statement for observational research (von Elm et al. 2008).
The local Research Ethics Committee assessed the study protocol and waived the need for informed consent (UMC Utrecht Medical Research Ethics Committee 19-029/C).

\section{Patients}

This retrospective observational study included patients aged $\geq 60$ years with postoperative elevated troponin levels as detected by routine troponin monitoring within the first 3 days after noncardiac surgery between January 1, 2017, and December 31, 2018, at the University Medical Center Utrecht, a tertiary referral hospital. Patients admitted immediately after the procedure at the intensive care unit (ICU) or the cardiac care unit (CCU) for more than 2 days were not visited by the dedicated anesthesiologists and were therefore excluded from the analysis because follow-up in these patients was conducted by intensivists or cardiologists as part of our local protocol. Patients who died within $24 \mathrm{~h}$ or in whom further therapy was withheld directly after surgery were also excluded. Patients, who underwent surgery more than once within the study period, were included as a new case. However, in case the procedure was performed within 3 days after the previous procedure, only the first procedure was included in the analysis.

\section{Postoperative care}

According to our local postoperative care protocol, cardiac troponin I (TnI) is measured in all noncardiac surgical patients aged $\geq 60$ years once daily on the first three postoperative days during hospital admission. This protocol excludes ophthalmic and plastic surgery patients because of low risk of cardiac complications. Troponin elevation was defined as TnI above the clinical cutoff level, which is the lowest value measurable with a $10 \%$ coefficient of variation above the 99th percentile upper reference limit (Thygesen et al. 2018). Two different TnI assays were used over the years. This resulted in a clinical cutoff of $\mathrm{TnI} \geq 60 \mathrm{ng} \mathrm{\textrm {L } ^ { - 1 }}$ (AccuTnI assay, Beckman Coulter, Brea, California, USA) from January 1, 2017, until May 16, 2018, and a clinical cutoff of highsensitive TnI $\geq 18 \mathrm{ng} \mathrm{L}^{-1}$ (Unicel DxI 800, Beckman Coulter, Brea, California, USA) from May 17, 2018, until December 31, 2018.

Patients with elevated TnI were consulted within the first three postoperative days, and longer if indicated. Management of these patients was left to the judgement 
of the attending anesthesiologist, which was based on a local protocol. This protocol advises to optimize myocardial oxygen supply and demand, and to conduct follow-up of troponin and an electrocardiogram (ECG) in patients with $\mathrm{TnI}>120 \mathrm{ng} \mathrm{L}^{-1}$ in case of no evident non-ischemic cause (e.g., sepsis, stress-induced cardiomyopathy) to rule out myocardial ischemia. This cutoff was based on two times the clinical cutoff of the assay used at that moment. Further, this protocol advises to consider consultation of a cardiologist, follow-up at the outpatient cardiac clinic, and prescription of antiplatelet therapy, statin, or beta-blockade. In patients with troponin $>600 \mathrm{ng} \mathrm{L}^{-1}$, i.e., more than ten times the clinical cutoff, a cardiology consultation is always recommended. Patients with mild troponin elevation were per protocol frequently not visited physically by the anesthesiologist because of a low risk of serious complications but were only followed up by TnI and ECG. A team of ten dedicated anesthesiologists with a particular interest in perioperative medicine were educated on the protocol.

\section{Data collection}

All data were collected from electronic medical files. These data included patient characteristics, comorbidities, revised cardiac risk index (RCRI) (Lee et al. 1999), American Society of Anesthesiologists (ASA) physical status classification (American Society of Anesthesiologists 2014), metabolic equivalent task score (METs) (Kristensen et al. 2014), and surgical risk as defined by the RCRI, and by the European Society of Cardiology (ESC) and the European Society of Anaesthesiology (ESA) (Kristensen et al. 2014). Additionally, data on visits by the dedicated anesthesiologist, diagnostics, inhospital postoperative complications, severity of complications according to the Clavien-Dindo classification (Dindo et al. 2004; Jammer et al. 2015), and in-hospital mortality were collected.

\section{Outcome}

The primary outcome was early detected (i.e., $\leq 7$ days after surgery) myocardial infarction as a result of consultation by the dedicated anesthesiologist. Myocardial infarction was defined as clinically diagnosed by the attending cardiologist. Secondary outcomes were overall detected complications, additional diagnostic tests advised by the dedicated anesthesiologist, treatment advised by the anesthesiologist, consultation of another medical specialist, and advised postoperative follow-up at the outpatient cardiac clinic. Potentially, a cardiologist could already have been consulted by the ward physician in patients with an elevated troponin or other cardiac complications, as the ward physicians were aware of the protocol. Therefore, we also recorded whether a cardiologist was consulted prior to or simultaneously with the visit by the dedicated anesthesiologists.

In addition to myocardial infarction, we assessed the occurrence of the following complications within 7 days after the procedure: arrhythmia diagnosed on 12-leads ECG or cardiac monitor, cardiopulmonary resuscitation, cerebrovascular accident (defined as radiologically confirmed ischemic or hemorrhagic stroke or transient ischemic attack), radiologically confirmed deep venous thrombosis and pulmonary embolism, sepsis as clinically diagnosed by the treating physician, pneumonia requiring antibiotics, respiratory failure requiring $\mathrm{MCU}$ (medium care unit) or ICU admission, acute kidney injury (AKI) defined as an increase in creatinine of $26.4 \mu \mathrm{mol} \mathrm{L} \mathrm{L}^{-1}$ or $25 \%$ from the preoperative creatinine value (Jammer et al. 2015), anemia defined as hemoglobin $<6.0 \mathrm{mmol} \mathrm{L}^{-1}\left(10 \mathrm{~g} \mathrm{dL}^{-1}\right.$, according to the Dutch guideline on blood transfusion(CBO 2011)), unexpected MCU or ICU admission, and unplanned reoperations. Additionally, length of hospital stay, mortality within 7 days, and the cause of death were assessed. Last, the Clavien-Dindo grade of the most severe complication within a week after surgery was recorded. A severe complication was defined as a Clavien-Dindo grade $\geq 3$ as this involves complications requiring a surgical, endoscopic, or radiologic intervention; life threatening complications requiring care in a high-dependency or intensive care unit; or death. (Dindo et al. 2004; Jammer et al. 2015) Additionally, we assessed the final suspected etiology of the elevated troponin as proposed by the dedicated anesthesiologist.

\section{Statistical analyses}

Baseline characteristics were compared dependent on the amount of troponin elevation (i.e., TnI 18-119 ng $\mathrm{L}^{-1}$, TnI 120-599 $\mathrm{ng} \mathrm{L}^{-1}$, and $\mathrm{TnI} \geq 600 \mathrm{ng} \mathrm{L}^{-1}$ ). These thresholds were chosen based on the thresholds as defined in our local protocol. The contribution of the dedicated anesthesiologist on the early detection of complications was assessed relative to the total number of complications. In addition, complications by their severity, length of stay, unexpected ICU or MCU admission, and death were assessed. The consultation rates by the dedicated anesthesiologists were evaluated, as were the number of ordered diagnostics, consulted medical specialties, and advised therapies. In order to determine whether care utility and complications were dependent on the height of troponin elevation, these were evaluated in subgroups of patients with different levels of troponin (i.e., TnI 18-119 $\mathrm{ng} \mathrm{L}^{-1}$, TnI 120$599 \mathrm{ng} \mathrm{L}^{-1}$, and $\mathrm{TnI} \geq 600 \mathrm{ng} \mathrm{L}^{-1}$ ).

Hemoglobin and creatinine measurements were missing in 103 and 204 patients, respectively. Since these variables were only used for descriptive statistics, we did 
not consider this an important source of bias and we did not impute the data. The statistical analyses were performed with R (Version 3.5.1-@ 2018-07-02, R, Inc., for Windows) (R Core Team (2016)).

\section{Results}

Within the study period, 8924 patients underwent noncardiac surgery of whom 1007 patients (11.2\%) had troponin elevation. Of these patients, 811 were eligible for inclusion (Fig. 1). Reason of exclusion was most often due to ICU admission for $>2$ days $(n=122)$. Troponin was mildly elevated, i.e., $18-119 \mathrm{ng} \mathrm{L}^{-1}$ in 543 patients (67\%); moderately elevated, i.e., $120-600 \mathrm{ng} \mathrm{L}^{-1}$ in 192 patients (24\%); and highly elevated, i.e., $\geq 600 \mathrm{ng}$ $\mathrm{L}^{-1}$ in 77 patients $(9 \%)$.

Baseline characteristics are reported in Table 1 . The median age was 74 years [IQR $68-80$ ], and $62 \%$ of the patients were males. Most patients underwent general $(28 \%)$ or vascular $(24 \%)$ surgery, and surgery was emergent in $40 \%$ of the patients. There were more patients with a history of ischemic heart disease and peripheral vascular disease in the group with highly elevated troponin compared to the other two categories.

\section{Postoperative outcomes}

A total of 804 complications occurred in 462 patients within 7 days after surgery (Table 2). The involvement of the dedicated anesthesiologist led to the diagnosis of 97
(12\%) of these complications in 63 patients (13\%). Postoperative complications were identified by the anesthesiologist in 18 patients (5\%) with a TnI 18-119 ng L ${ }^{-1}, 21$ patients (13\%) with a TnI $120-599 \mathrm{ng} \mathrm{L}^{-1}$, and 24 patients $(33 \%)$ with a $\mathrm{TnI} \geq 600 \mathrm{ng} \mathrm{L}^{-1}$. The anesthesiologists were involved in the discovery of 19 (59\%) of 32 postoperative myocardial infarctions within 1 week after surgery. Besides cardiac ischemia, 76 (9.4\%) patients developed an arrhythmia, but patients were also often diagnosed with noncardiac complications, including respiratory failure (8.9\%), pneumonia (13.2\%), and acute kidney injury (17.5\%). Patients with higher troponin levels suffered from more complications, had more often an unexpected ICU or MCU admission, and had a longer median hospital length of stay. Ten patients (1\%) died in the first week after surgery, and 251 patients (31\%) had a complication graded as Clavien-Dindo $\geq 3$ (Table 2).

\section{Postoperative consultation}

A total of 509 patients (63\%) received consultation by a dedicated anesthesiologist (Table 3). In 35 patients (7\%), the anesthesiologists provided advice as recorded in the electronic medical file, without an actual physical visit. In patients who experienced at least one complication ( $n$ = 462), 161 patients (53\%) were not visited by the dedicated anesthesiologist; in 18 patients (51\%), only advice was provided without an actual physical visit by the anesthesiologist, 60 (59\%) patients were visited once,

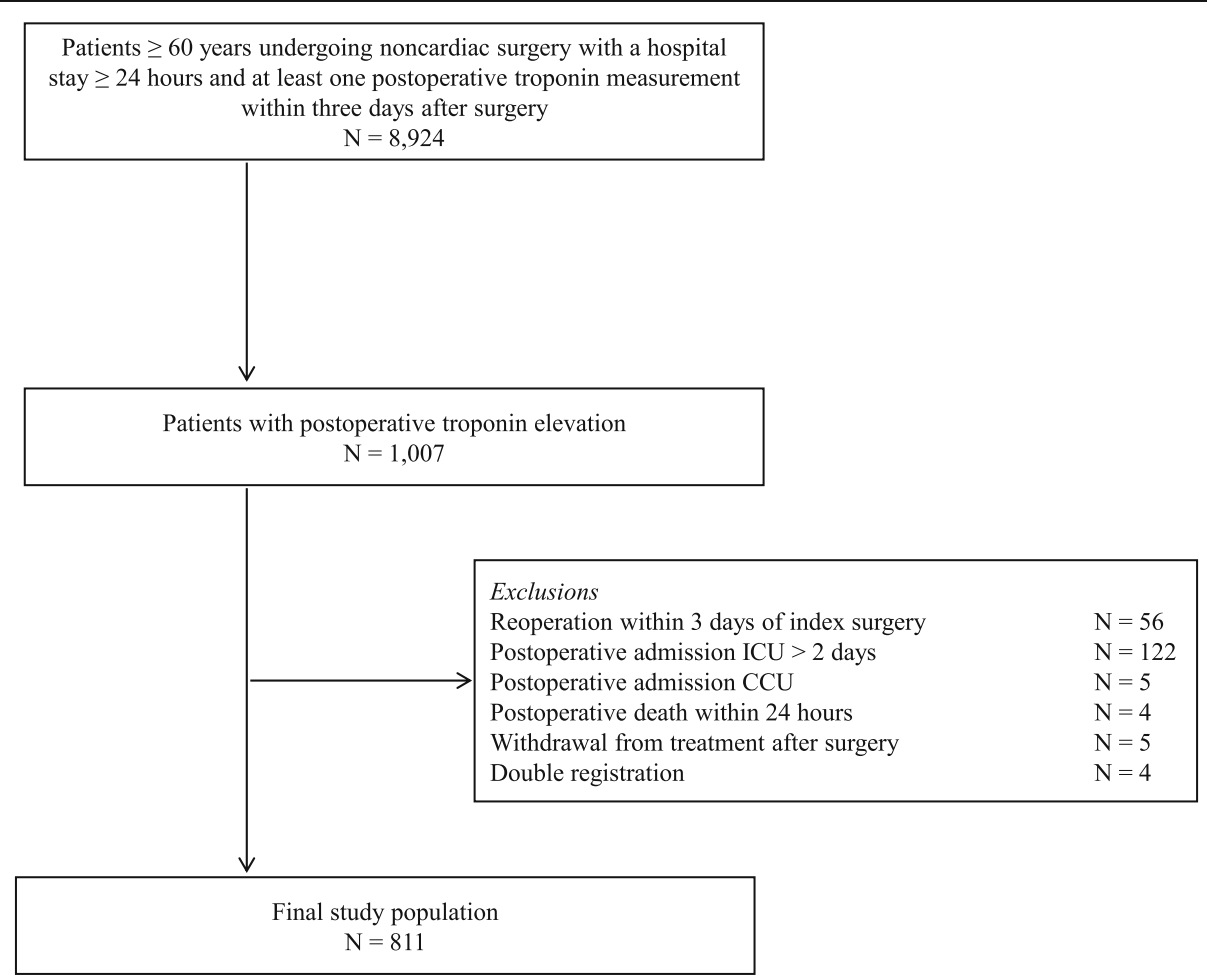

Fig. 1 Patient inclusion. ICU, intensive care unit; CCU, cardiac care unit 
Table 1 Baseline characteristics dependent on the height of troponin elevation

\begin{tabular}{|c|c|c|c|c|}
\hline & Overall & $\begin{array}{l}\text { Tnl } \\
18-119 \mathrm{ng} \mathrm{L}^{-1}\end{array}$ & $\begin{array}{l}\text { Tnl } \\
120-599 \mathrm{ng} \mathrm{L}^{-1}\end{array}$ & $\mathrm{Tnl} \geq 600 \mathrm{ng} \mathrm{L}^{-1}$ \\
\hline N & 811 & 543 & 192 & 76 \\
\hline Age (median [IQR]) & $74[68-80]$ & $74[68-80]$ & 74 [68-79] & $73[69-79]$ \\
\hline Males (\%) & $505(62.3)$ & $341(62.8)$ & $115(59.9)$ & $49(64.5)$ \\
\hline \multicolumn{5}{|l|}{ RCRI score (\%) } \\
\hline 0 & $196(24.2)$ & $141(26.0)$ & $45(23.4)$ & $10(13.2)$ \\
\hline 1 & $320(39.5)$ & 215 (39.6) & $77(40.1)$ & $28(36.8)$ \\
\hline 2 & 191(23.6) & $120(22.1)$ & $45(23.4)$ & $26(34.2)$ \\
\hline 3 or more & $104(12.8)$ & $67(12.3)$ & $25(13.0)$ & $12(15.8)$ \\
\hline High-risk surgery (defined by RCRI) (\%) & $314(38.7)$ & $195(35.9)$ & $82(42.7)$ & $37(48.7)$ \\
\hline High-risk surgery (defined by ESC/ESA) (\%) & $124(15.3)$ & $77(14.2)$ & $35(18.2)$ & $12(15.8)$ \\
\hline History of ischemic heart disease (\%) & $235(29.0)$ & $145(26.7)$ & $58(30.2)$ & $32(42.1)$ \\
\hline History of congestive heart failure (\%) & $84(10.4)$ & $60(11.0)$ & $19(9.9)$ & $5(6.6)$ \\
\hline History of cerebrovascular disease (\%) & $201(24.8)$ & $132(24.3)$ & $48(25.0)$ & $21(27.6)$ \\
\hline Insulin dependent diabetes (\%) & 109 (13.4) & $74(13.6)$ & $24(12.5)$ & $11(14.5)$ \\
\hline $\begin{array}{l}\text { Preoperative creatinine } \\
>2.0 \mathrm{mg} \mathrm{dL}^{-1}(\%)\end{array}$ & $113(13.9)$ & $83(15.3)$ & $18(9.4)$ & $12(15.8)$ \\
\hline Arrhythmia (\%) & $185(22.8)$ & $136(25.0)$ & $42(21.9)$ & $7(9.2)$ \\
\hline ICD or pacemaker (\%) & $65(8.0)$ & $53(9.8)$ & $10(5.2)$ & $2(2.6)$ \\
\hline Valvular disease (\%) & $113(13.9)$ & $82(15.1)$ & $23(12.0)$ & $8(10.5)$ \\
\hline $\begin{array}{l}\text { Peripheral vascular } \\
\text { disease (\%) }\end{array}$ & $241(29.7)$ & $140(25.8)$ & $66(34.4)$ & $35(46.1)$ \\
\hline Hypertension (\%) & $516(63.6)$ & $347(63.9)$ & $119(62.0)$ & $50(65.8)$ \\
\hline Pulmonary disease (\%) & $210(25.9)$ & $142(26.2)$ & $54(28.1)$ & $14(18.4)$ \\
\hline Active malignancy (\%) & $255(31.4)$ & $172(31.7)$ & $60(31.2)$ & $23(30.3)$ \\
\hline Renal failure (\%) & $282(34.8)$ & $194(35.7)$ & $58(30.2)$ & $30(39.5)$ \\
\hline Diabetes mellitus (\%) & $189(23.3)$ & $129(23.8)$ & $41(21.4)$ & $19(25.0)$ \\
\hline \multicolumn{5}{|l|}{ ASA physical status (\%) } \\
\hline 1 & $4(0.5)$ & $3(0.6)$ & $0(0.0)$ & $1(1.3)$ \\
\hline 2 & $229(28.2)$ & $161(29.7)$ & $53(27.6)$ & $15(19.7)$ \\
\hline 3 & $481(59.3)$ & $319(58.7)$ & $112(58.3)$ & $50(65.8)$ \\
\hline 4 & $88(10.9)$ & $55(10.1)$ & $25(13.0)$ & $8(10.5)$ \\
\hline 5 & $9(1.1)$ & $5(0.9)$ & $2(1.0)$ & $2(2.6)$ \\
\hline \multicolumn{5}{|l|}{ METS (\%) } \\
\hline 1-3 METS & $224(27.6)$ & $162(29.8)$ & $42(21.9)$ & $20(26.3)$ \\
\hline 4-7 METS & $373(46.0)$ & $263(48.4)$ & $79(41.1)$ & $31(40.8)$ \\
\hline 8-10 METS & $21(2.6)$ & $10(1.8)$ & $9(4.7)$ & $2(2.6)$ \\
\hline Unknown & $193(23.8)$ & $108(19.9)$ & $62(32.3)$ & $23(30.3)$ \\
\hline Emergency surgery (\%) & $328(40.4)$ & $213(39.2)$ & $83(43.2)$ & $32(42.1)$ \\
\hline \multicolumn{5}{|l|}{ Surgical specialty (\%) } \\
\hline General & $223(27.5)$ & $149(27.4)$ & $58(30.2)$ & $16(21.1)$ \\
\hline Gynecological & $25(3.1)$ & $18(3.3)$ & $5(2.6)$ & $2(2.6)$ \\
\hline Head and Neck & $78(9.6)$ & $54(9.9)$ & $15(7.8)$ & $9(11.8)$ \\
\hline Neurological & $133(16.4)$ & $94(17.3)$ & $28(14.6)$ & $11(14.5)$ \\
\hline Orthopedic & $104(12.8)$ & $71(13.1)$ & $26(13.5)$ & $7(9.2)$ \\
\hline
\end{tabular}


Table 1 Baseline characteristics dependent on the height of troponin elevation (Continued)

\begin{tabular}{|c|c|c|c|c|}
\hline & Overall & $\begin{array}{l}\text { Tnl } \\
18-119 \mathrm{ng} \mathrm{L}^{-1}\end{array}$ & $\begin{array}{l}\text { Tnl } \\
120-599 \mathrm{ng} \mathrm{L}^{-1}\end{array}$ & $\mathrm{Tnl} \geq 600 \mathrm{ng} \mathrm{L}^{-1}$ \\
\hline N & 811 & 543 & 192 & 76 \\
\hline Urological & $57(7.0)$ & $42(7.7)$ & $9(4.7)$ & $6(7.9)$ \\
\hline Vascular & $191(23.6)$ & $115(21.2)$ & $51(26.6)$ & $25(32.9)$ \\
\hline Locoregional and neuraxial anesthesia (\%) & $62(7.6)$ & $45(8.3)$ & $13(6.8)$ & $4(5.3)$ \\
\hline
\end{tabular}

Troponin thresholds were chosen based on the thresholds as defined in the local protocol of the University Medical Center Utrecht (Tnl 18-119 ng L ${ }^{-1}$, Tnl 120$599 \mathrm{ng} \mathrm{L}^{-1}$, and $\mathrm{Tnl} \geq 600 \mathrm{ng} \mathrm{L}^{-1}$ ). Ischemic heart disease was defined as a history of myocardial infarction or previous revascularization, and a history of congestive heart failure was defined as an estimated left ventricular ejection fraction $<40 \%$. Cerebrovascular disease was defined as a history of ischemic stroke, hemorrhagic stroke, or transient ischemic attacks. Renal failure was defined as a glomerular filtration rate (GFR) $<60 \mathrm{ml} \mathrm{min}^{-1}$ in the last 3 months) ASA American Society of Anesthesiologists classification, ESA European Society of Anaesthesiology, ESC European Society of Cardiology, ICD implantable cardioverter defibrillator, IQR interquartile range, METs metabolic equivalent of task score, $R C R I$ revised cardiac risk index, $T n /$ troponin I

Table 2 Postoperative outcomes dependent on the height of troponin elevation

\begin{tabular}{|c|c|c|c|c|c|c|}
\hline \multirow[b]{2}{*}{$\mathrm{N}$} & \multicolumn{2}{|c|}{ Tnl 18-119 ng L ${ }^{-1}$} & \multicolumn{2}{|c|}{ Tnl 120-599 ng L-1 } & \multicolumn{2}{|c|}{$\mathrm{Tnl} \geq 600 \mathrm{ng} \mathrm{L}^{-1}$} \\
\hline & 543 & & 192 & & 76 & \\
\hline Complications $\leq 7$ days & Total & By $\mathrm{POA}^{\mathrm{C}}$ & Total & By $\mathrm{POA}^{\mathrm{C}}$ & Total & By $\mathrm{POA}^{\mathrm{c}}$ \\
\hline Myocardial infarction (\%) & $1(0.2)$ & $1(0.2)$ & $8(4.2)$ & $2(1.0)$ & $23(30.3)$ & $16(21.1)$ \\
\hline Arrhythmia (\%) & $46(8.5)$ & $3(0.6)$ & $22(11.5)$ & $0(0.0)$ & $9(11.8)$ & $3(3.9)$ \\
\hline CPR (\%) & $0(0.0)$ & $0(0.0)$ & $2(1.0)$ & $0(0.0)$ & $2(2.6)$ & $0(0.0)$ \\
\hline Cerebrovascular accident (\%) & $9(1.7)$ & $0(0.0)$ & $2(1.0)$ & $0(0.0)$ & $3(3.9)$ & $0(0.0)$ \\
\hline Deep venous thrombosis (\%) & $4(0.7)$ & $0(0.0)$ & $0(0.0)$ & $0(0.0)$ & $0(0.0)$ & $0(0.0)$ \\
\hline Pulmonary embolism (\%) & $10(1.8)$ & $0(0.0)$ & $5(2.6)$ & $0(0.0)$ & $3(3.9)$ & $1(1.3)$ \\
\hline Sepsis (\%) & $20(3.7)$ & $0(0.0)$ & $8(4.2)$ & $1(0.5)$ & $5(6.6)$ & $1(1.3)$ \\
\hline Pneumonia (\%) & $61(11.2)$ & $6(1.1)$ & $31(16.1)$ & $0(0.0)$ & 15 (19.7) & $2(2.6)$ \\
\hline Respiratory failure (\%) & $33(6.1)$ & $1(0.2)$ & $25(13.0)$ & $6(3.1)$ & $14(18.4)$ & $1(1.3)$ \\
\hline Acute kidney injury ${ }^{a}(\%)$ & $59(15.1)$ & $1(0.0)$ & $33(21.7)$ & $0(0.0)$ & $14(22.2)$ & $4(14.8)$ \\
\hline Anemia $^{\mathrm{b}}(\%)$ & $198(36.5)$ & $6(6.6)$ & $87(45.3)$ & $28(14.6)$ & $42(55.3)$ & $14(18.4)$ \\
\hline \multicolumn{7}{|l|}{ Clavien-Dindo Classification (\%) } \\
\hline Grade 1 & $73(13.4)$ & & $16(8.3)$ & & $5(6.6)$ & \\
\hline Grade 2 & 206 (37.9) & & $67(34.9)$ & & $25(32.9)$ & \\
\hline Grade 3a & $11(2.0)$ & & $10(5.2)$ & & $1(1.3)$ & \\
\hline Grade 3b & $26(4.8)$ & & $9(4.7)$ & & $2(2.6)$ & \\
\hline Grade 4a & 74 (13.6) & & $47(24.5)$ & & $27(35.5)$ & \\
\hline Grade 4b & $19(3.5)$ & & $7(3.6)$ & & $8(10.5)$ & \\
\hline Grade 5 & $5(0.9)$ & & $4(2.1)$ & & $1(1.3)$ & \\
\hline No complications & $129(23.8)$ & & $32(16.7)$ & & $7(9.2)$ & \\
\hline Unexpected ICU admission (\%) & $21(3.9)$ & & $13(6.8)$ & & $11(14.5)$ & \\
\hline Unexpected MCU admission (\%) & $91(16.8)$ & & $5(28.6)$ & & $33(43.4)$ & \\
\hline Reoperation (\%) & $50(9.2)$ & & $19(9.9)$ & & $8(10.5)$ & \\
\hline Length of stay (median [IQR]) & 7 [4-15] & & $9[5-16]$ & & $11[6-16]$ & \\
\hline Mortality within 1 week (\%) & $5(0.9)$ & & $4(2.1)$ & & $1(1.3)$ & \\
\hline
\end{tabular}

Troponin thresholds were chosen based on the thresholds as defined in the local protocol of the University Medical Center Utrecht (Tnl 18-119 $\mathrm{ng} \mathrm{L}^{-1}$, Tnl 120$599 \mathrm{ng} \mathrm{L}^{-1}$, and $\mathrm{Tnl} \geq 600 \mathrm{ng} \mathrm{L}^{-1}$ )

$C P R$ cardiopulmonary resuscitation, ICU intensive care unit, $I Q R$ interquartile range, $M C U$ medium care unit, NA not applicable, TnI troponin I

${ }^{a}$ Acute kidney injury (AKI) was calculated based on the KDIGO criteria. (1) AKI was based on 607 patients as no pre- and/or postoperative creatinine was available in 204 patients (25\%)

${ }^{\mathrm{b}}$ Anemia was defined as a hemoglobin $<6.0 \mathrm{mmol} \mathrm{L}^{-1}\left(10 \mathrm{~g} \mathrm{dL}^{-1}\right)$, which is based on 708 patients as hemoglobin was not measured in 103 patients $(13 \%)$

${ }^{\mathrm{C}}$ Early detection of complication (within 3 days) through contribution of the dedicated team of anesthesiologist (POA: perioperative anesthesiologist) 
Table 3 Consultations, interventions, and causes of troponin elevation assigned by the dedicated anesthesiologist, dependent on the height of troponin elevation

\begin{tabular}{|c|c|c|c|}
\hline & Tnl 18-119 ng L ${ }^{-1}$ & Tnl 120-599 ng L ${ }^{-1}$ & $\mathrm{Tnl} \geq 600 \mathrm{ng} \mathrm{L}^{-1}$ \\
\hline N & 543 & 192 & 76 \\
\hline \multicolumn{4}{|l|}{ Consultation (\%) } \\
\hline No visit, advice only & $28(5.2)$ & $4(2.1)$ & $3(3.9)$ \\
\hline Only 1-time visit & $186(34.3)$ & $90(46.9)$ & $39(51.3)$ \\
\hline$>1$ visit & $88(16.2)$ & $49(25.5)$ & $22(28.9)$ \\
\hline Any diagnostics ordered (\%) & $207(38.1)$ & $126(65.6)$ & $48(63.2)$ \\
\hline ECG & $201(37.0)$ & $120(62.5)$ & $47(61.8)$ \\
\hline Echocardiography & $3(0.6)$ & $19(9.9)$ & $26(34.2)$ \\
\hline $\mathrm{CT}$ angiography & $2(0.4)$ & $3(1.6)$ & $4(5.3)$ \\
\hline Additional troponin & $76(14.0)$ & $61(31.8)$ & $38(50.0)$ \\
\hline Other enzymes (i.e., CK-MB) & $23(4.2)$ & $43(22.4)$ & $36(47.4)$ \\
\hline \multicolumn{4}{|l|}{ Other specialty consulted (\%) } \\
\hline Cardiology & $39(7.2)$ & $65(33.9)$ & $51(67.1)$ \\
\hline Pulmonology & $5(0.9)$ & $2(1.0)$ & $0(0.0)$ \\
\hline Other $^{a}$ & $6(1.1)$ & $2(1.0)$ & $0(0.0)$ \\
\hline Cardiology already involved (\%) & $57(10.5)$ & $46(24.0)$ & $21(27.6)$ \\
\hline Any change in treatment (\%) & $55(10.1)$ & $47(24.5)$ & $29(38.2)$ \\
\hline Change in medication & $24(4.4)$ & $26(13.5)$ & $24(31.6)$ \\
\hline Red blood cell transfusion & $6(6.6)$ & $28(14.6)$ & $14(18.4)$ \\
\hline Follow up at outpatient cardiac clinic (\%) & $73(13.4)$ & $64(33.3)$ & $50(65.8)$ \\
\hline \multicolumn{4}{|l|}{ Cause of myocardial injury (\%) } \\
\hline Ischemic heart disease & $6(1.1)$ & $12(6.2)$ & $28(36.8)$ \\
\hline Arrhythmia & $22(4.1)$ & $8(4.2)$ & $0(0.0)$ \\
\hline Congestive heart failure & $6(1.1)$ & $2(1.0)$ & $3(3.9)$ \\
\hline Pulmonary embolism & $4(0.7)$ & $1(0.5)$ & $2(2.6)$ \\
\hline Pneumonia & $7(1.3)$ & $4(2.1)$ & $0(0.0)$ \\
\hline Respiratory failure & $11(2.0)$ & $9(4.7)$ & $1(1.3)$ \\
\hline Sepsis & $9(1.7)$ & $6(3.1)$ & $2(2.6)$ \\
\hline Acute kidney injury & $31(5.7)$ & $6(3.1)$ & $0(0.0)$ \\
\hline Anemia & $15(2.8)$ & $8(4.2)$ & $4(5.3)$ \\
\hline Fluid overload & $4(0.7)$ & $4(2.1)$ & $0(0.0)$ \\
\hline Hypertension & $6(1.1)$ & $3(1.6)$ & $0(0.0)$ \\
\hline Perioperative hemodynamics ${ }^{\mathrm{b}}$ & 66 (12.2) & $37(19.3)$ & $9(11.8)$ \\
\hline Other ${ }^{c}$ & $15(2.8)$ & $8(4.2)$ & $3(3.9)$ \\
\hline Cause unknown & $341(62.8)$ & $84(43.8)$ & 24 (31.6) \\
\hline
\end{tabular}

Troponin thresholds were chosen based on the thresholds as defined in the local protocol of the University Medical Center Utrecht (Tnl 18-119 ng L ${ }^{-1}$, Tnl 120$599 \mathrm{ng} \mathrm{L}^{-1}$, and $\mathrm{Tnl} \geq 600 \mathrm{ng} \mathrm{L}^{-1}$ )

alncluding nephrologist, hematologist, cardiothoracic surgeon, and geriatrician

${ }^{\mathrm{b}}$ Including hypotension and tachycardia

Including pericarditis, myocardial contusion, neurological conditions (e.g., subarachnoid hemorrhage), and fever

ECG electrocardiogram, CT computed tomography, CK-MB creatine-kinase isoenzyme, MCU surgical medium care unit, PACU post anesthesia care unit, Tnl troponin I

and $99(62 \%)$ patients were visited more than once. In 124 patients $(24 \%)$, the cardiologist was already involved in patient care prior to or simultaneously with the dedicated anesthesiologist, and the cardiologist was consulted by the dedicated anesthesiologist in 155 patients $(30 \%)$. In total, 187 patients $(23 \%)$ were referred to the cardiac outpatient clinic for further follow-up after discharge. 
There were more consultations by the dedicated anesthesiologists in patients with $\mathrm{TnI} \geq 600 \mathrm{ng} \mathrm{L}^{-1}$ compared with the other two categories. In addition, a cardiologist was more frequently consulted ( $80 \%$ vs. $45 \%$ and $13 \%$ for patients with $\mathrm{TnI} \geq 600 \mathrm{ng} \mathrm{L}^{-1}$ vs. TnI $120-599 \mathrm{ng}$ $\mathrm{L}^{-1}$ and TnI $18-120 \mathrm{ng} \mathrm{L}^{-1}$, respectively) or already involved (33\% vs. $32 \%$ and $19 \%$, respectively) (Table 3).

Additional diagnostics were ordered by the anesthesiologist in 381 patients (75\%). An ECG was most frequently ordered (73\%), followed by additional troponin (34\%) and other cardiac enzymes (20\%). A change in treatment (i.e., a change in medication or red blood cell transfusion) was initiated by the anesthesiologist in 131 patients (16\%), with 48 patients receiving blood products (6\%). There were more diagnostic tests ordered and treatments initiated by the dedicated anesthesiologist in patients with higher troponin levels (Table 3).

In 449 (55\%) patients, no clear cause of the troponin elevation was identified (Table 3). In $22 \%$ of patients, it was considered to be caused by unstable perioperative hemodynamics, including hypotension and tachycardia, followed by ischemic heart disease (9\%) and AKI (7\%).

\section{Discussion}

This study evaluated the contribution of follow-up by dedicated anesthesiologists on postoperative care utility and early detection of complications in patients with elevated troponin after noncardiac surgery. The anesthesiologists were primarily responsible for the early detection of $12 \%$ of all postoperative complications within 1 week after surgery, with an especially large contribution (59\%) to detection of myocardial infarctions.

\section{Literature}

The current study found that routine postoperative troponin surveillance, supported by routine postoperative consultations by a dedicated anesthesiologist, can be used to detect postoperative complications at an early stage. Increasing rates of both cardiovascular, i.e., myocardial infarction and pulmonary embolism, and noncardiac complications, i.e., sepsis, respiratory failure, renal failure, and anemia, were found in patients with elevated troponin in a dose-dependent manner. Substantially more patients with postoperative troponin elevation were consulted after implementation of a dedicated anesthesia team to conduct these visits $(41 \%$ were consulted by cardiologists in the study by Van Waes et al. (2016) versus $63 \%$ in the current study).

Around $15 \%$ of patients with elevated troponin received a change in medication, which is in accordance with a previous study (van Waes et al. 2016). Six percent received red blood cell transfusion after involvement of the dedicated anesthesiologist, aiming at a hemoglobin level $>6 \mathrm{mmol} \mathrm{L}^{-1}\left(10 \mathrm{~g} \mathrm{dL}^{-1}\right)$. Interestingly, a previous study found that the cardiologist only advised red blood cell transfusion in $2 \%$ of patients with postoperative troponin elevation (van Waes et al. 2016).

\section{Clinical implications}

Prediction of the risk of postoperative complications has been shown to be difficult using only preoperative parameters (Wijeysundera 2016). Although preoperative patient optimization and planning of perioperative care might have a larger effect on clinical outcome and costs than postoperative visits by trained anesthesiologist solely (Dexter and Wachtel 2014), we do believe there may be an additive beneficial effect provided by these visits. Ideally, one might argue that anesthesiologists should consult all patients receiving intermediate or high-risk surgery, but resources are scarce. By identifying patients at risk not only before surgery but again in the postoperative phase using additional data, resources can be directed towards where needed most. Troponin surveillance may be an efficient manner to conduct this postoperative selection (Devereaux et al. 2017; van Waes et al. 2016; Weber et al. 2013). Therefore, we focused on patients with elevated troponins to select those at risk of postoperative complications and to first determine the effect of this intervention, before unrolling it to a broader patient population.

The present study found that follow-up by dedicated anesthesiologists enabled early detection of $12 \%$ of all complications and $59 \%$ of all postoperative myocardial infarctions within 1 week after surgery. The experience of anesthesiologists with procedure-related complications in addition to their knowledge of the cardiopulmonary and central nervous system can provide a valuable contribution to postoperative care by surgeons and care by cardiologists in case of postoperative troponin elevation. In our institution, the resources needed for a dedicated team of anesthesiologists conducting postoperative visits in this group of patients are low. One dedicated anesthesiologist on call spends on average $1 \mathrm{~h}$ per day on screening and follow-up of patients with postoperative troponin elevation. In case of any emergent complications caught by this anesthesiologist, additional time is needed for extra diagnostics, consultation of other medical specialties, and follow-up. The remaining time is spent on clinical tasks at the outpatient clinic not evaluated in this study, such as preoperative assessment of patients and multidisciplinary meetings. In our center, no extra personnel needed to be employed to implement this program. In addition, a cardiologist was consulted in only $30 \%$ of patients instead of $41 \%$ as reported previously (van Waes et al. 2016). Although additional diagnostic procedures were performed, these were mostly low-cost such as an ECG, while the number of patients in whom a complication was diagnosed and medical 
treatment was changed, was substantial. Importantly, in a majority of the patients (55\%) a clear cause of troponin elevation could not be identified. Therefore, treatment options currently are often limited. Prospective studies are necessary to further determine the potential effect of postoperative visits by dedicated anesthesiologist on patient outcome.

\section{Strengths and limitations}

To the best of our knowledge, this is the first study reporting on the effect of postoperative anesthesia visits on resource utility and detection of postoperative complications in patients with postoperative troponin elevation. It was conducted in the non-cardiac surgery population, making these results generalizable to a large group of patients.

This study has some important limitations. First, because of the retrospective design of the study and limited number of patients, we were unable to estimate whether the implementation of routine postoperative anesthesia visits resulted in better patient outcomes. Second, two different troponin assays, i.e., normal TnI and the high sensitive TnI, were used during the inclusion period. As the second assay is more sensitive, this might have resulted in a higher incidence of troponin elevation. However, there were no differences in preoperative characteristics, but we did find more myocardial infarctions in the period the normal TnI assay was used together with more cardiology consultations, extra ordered diagnostics, and change in medication. Third, many patients were referred to the cardiac outpatient clinic for further follow-up, but often this was conducted in other institutions, since the UMC Utrecht is a tertiary referral hospital. This hampered our ability to track down diagnostics and interventions instituted during these follow-up visits. Fourth, there were some missing values for creatinine and hemoglobin. Incidence rates of postoperative anemia and AKI are probably overestimated as their markers were mostly missing in healthier patients. Fifth, because myocardial infarction was defined as a clinical diagnosis made by a cardiologist, the incidence of myocardial infarction may have been higher when it retrospectively would have been based on the 4th universal definition (Thygesen et al. 2018), as previously shown (Beattie et al. 2018; Devereaux et al. 2011; van Waes et al. 2016). Finally, only documented consultations by the anesthesiologist were recorded as consultation, which potentially explains why $37 \%$ of the patients did not receive a consultation. As part of the local protocol, anesthesiologists determined whether consultation was necessary based on clinical course reported in the electronic medical files and consultation of treating physicians by phone. We presume that in patients with elevated but low troponin levels (especially with TnI 19-120 ng/l), an ECG was remotely assessed by the anesthesiologist and discussed with the treating physician. In case of no abnormalities, nothing was documented in the electronical medical files, although in fact remote consultation did occur.

\section{Conclusion}

Implementation of routine postoperative consultations by dedicated anesthesiologists resulted in early detection and treatment of complications in patients with postoperative elevated troponin levels.

\section{Abbreviations \\ AKI: Acute kidney injury; ASA: American Society of Anesthesiologists; CCU: Cardiac care unit; CK-MB: Creatine-kinase isoenzyme; \\ CPR: Cardiopulmonary resuscitation; CT: Computed tomography; ECG: Electrocardiogram; ESA: European Society of Anaesthesiology; ESC: European Society of Cardiology; GFR: Glomerular filtration rate; ICD: Implantable cardioverter defibrillator; ICU: Intensive care unit; IQR: Interquartile range; MCU: Medium care unit; METs: Metabolic equivalent of task score; NA: Not applicable; PACU: Post anesthesia care unit; POA: Perioperative anesthesiologist; RCRI: Revised cardiac risk index; Tnl: Troponin I; UMC: University Medical Center}

\section{Acknowledgements \\ None}

\section{Authors' contributions}

Annemarie Akkermans, MD.: This author helped design the study, collect the data, perform the analysis, and wrote and edit the manuscript. Lisette $\mathrm{M}$. Vernooij, MSc: This author helped design the study, collect the data, perform the analysis, and wrote and edit the manuscript. Wilton A. van Klei, MD, PhD: This author helped design the study, wrote and edit the manuscript. Judith A. van Waes, MD, PhD: This author helped design the study and wrote and edit the manuscript. The author(s) read and approved the final manuscript.

\section{Funding}

None

Availability of data and materials

The datasets used and/or analyzed during the current study are available from the corresponding author on reasonable request.

Ethics approval and consent to participate

The local Research Ethics Committee assessed the study protocol and waived the need for informed consent (UMC Utrecht Medical Research Ethics Committee 19-029/C).

Consent for publication

Not applicable.

Competing interests

None

Received: 3 January 2020 Accepted: 10 June 2020

Published online: 16 July 2020

\section{References}

Akkermans A, Peelen LM, van Waes JA, Rinkel GJ, van Klei WA. Cardiac events within one year after a subarachnoid haemorrhage: the predictive value of troponin elevation after aneurysm occlusion. Eur J Prev Cardiol. 2019;26(4):420-8.

American Society of Anesthesiologists. ASA Physical Status Classification System. 2014.

Beattie WS, Wijeysundera DN, Chan MTV, Peyton PJ, Leslie K, Paech MJ, et al. Implication of major adverse postoperative events and myocardial injury on disability and survival. Anesth Analg. 2018;127(5):1118-26 Available from: http://www.ncbi.nlm.nih.gov/pubmed/29533264. [cited 2019 Jan 3].

CBO. Richtlijn Bloedtransfusie (Guideline blood transfusion); 2011. p. 182-3. Devereaux PJ, Biccard BM, Sigamani A, Xavier D, Chan MTV, Srinathan SK, et al. Association of postoperative high-sensitivity troponin levels with myocardial 
injury and 30-day mortality among patients undergoing noncardiac surgery JAMA. 2017;317(16):1642 Available from: http://www.ncbi.nlm.nih.gov/ pubmed/28444280. [cited 2017 May 23].

Devereaux PJ, Chan MTV, Alonso-Coello P, Walsh M, Berwanger O, Villar JC, et al. Association between postoperative troponin levels and 30-day mortality among patients undergoing noncardiac surgery. JAMA. 2012;307(21):2295-304 Available from: http.//www.ncbinlm.nih.gov/pubmed/22706835. [cited 2015 Jun 3].

Devereaux PJ, Xavier D, Pogue J, Guyatt G, Sigamani A, Garutti I, et al. Characteristics and short-term prognosis of perioperative myocardial infarction in patients undergoing noncardiac surgery: a cohort study. Ann Intern Med. 2011;154(8):523-8 Available from: http://www.ncbi.nlm.nih.gov/ pubmed/21502650. [cited 2015 Jun 3].

Dexter F, Wachtel RE. Strategies for net cost reductions with the expanded role and expertise of anesthesiologists in the perioperative surgical home. Anesth Analg. 2014;118(5):1062-71.

Dindo D, Demartines N, Clavien P-A. Classification of surgical complications. Ann Surg. 2004;240(2):205-13.

Duceppe E, Parlow J, MacDonald P, Lyons K, McMullen M, Srinathan S, et al. Canadian Cardiovascular Society Guidelines on perioperative cardiac risk assessment and management for patients who undergo noncardiac surgery. Can J Cardiol. 2017;33(1):17-32. Available from: http://www.ncbi.nlm.nih.gov/ pubmed/27865641. [cited 2018 Jan 23].

Jammer I, Wickboldt N, Sander M, Smith A, Schultz MJ, Pelosi P, et al. Standards for definitions and use of outcome measures for clinical effectiveness research in perioperative medicine: European Perioperative Clinical Outcome (EPCO) definitions: a statement from the ESA-ESICM joint taskforce on perioperative outcome measure. Eur J Anaesthesiol. 2015;32(2):88-105.

Kristensen SD, Knuuti J, Saraste A, Anker S, Bøtker HE, De Hert S, et al. 2014 ESC/ ESA Guidelines on non-cardiac surgery: cardiovascular assessment and management. Eur Heart J. 2014;35(35):2383-431 Available from: http://www. ncbi.nlm.nih.gov/pubmed/25086026. [cited 2016 Dec 19].

Lee TH, Marcantonio ER, Mangione CM, Thomas EJ, Polanczyk CA, Cook EF, et al. Derivation and prospective validation of a simple index for prediction of cardiac risk of major noncardiac surgery. Circulation. 1999;100(10):1043-9 Available from: http://www.ncbi.nlm.nih.gov/pubmed/10477528. [cited 2016 May 1].

Levy M, Heels-Ansdell D, Hiralal R, Bhandari M, Guyatt G, Yusuf S, et al. Prognostic value of troponin and creatine kinase muscle and brain isoenzyme measurement after noncardiac surgery: a systematic review and metaanalysis. Anesthesiology. 2011;114(4):796-806 Available from: http:// anesthesiology.pubs.asahq.org/Article.aspx?doi $=10.1097 /$ ALN . Ob013e31820ad503. [cited 2016 Dec 19].

Noordzij PG, Van Geffen O, Dijkstra IM, Boerma D, Meinders AJ, Rettig TCD, et al. High-sensitive cardiac troponin T measurements in prediction of non-cardiac complications after major abdominal surgery. Br J Anaesth. 2015;114(6):909-18.

R Core Team. R: A language and environment for statistical computing. Vienna: R Found. Stat. Comput; 2016.

Thygesen K, Alpert JS, Jaffe AS, et al. Fourth Universal Definition of Myocardial Infarction (2018). Circulation. 2018:138(20):e618-e651.

van Waes JAR, Grobben RB, Nathoe HM, Kemperman H, de Borst GJ, Peelen LM, et al. One-year mortality, causes of death, and cardiac interventions in patients with postoperative myocardial injury. Anesth Analg. 2016;123(1):29-37. Available from: http://www.ncbi.nlm.nih.gov/pubmed/27111647. [cited 2017 May 23].

van Waes JAR, Nathoe HM, de Graaff JC, Kemperman H, de Borst GJ, Peelen LM, et al. Myocardial injury after noncardiac surgery and its association with short-term mortality. Circulation. 2013;127(23):2264-71 Available from: http:// www.ncbi.nlm.nih.gov/pubmed/23667270. [cited 2015 May 27].

von Elm E, Altman D, Egger M, Pocock S, Gøtzsche P, Vandenbroucke J, STROBE Initiative. The Strengthening the Reporting of Observational Studies in Epidemiology (STROBE) statement: guidelines for reporting observational studies. J Clin Epidemiol. 2008;61(4):344-9.

Weber M, Luchner A, Manfred S, Mueller C, Liebetrau C, Schlitt A, et al. Incremental value of high-sensitive troponin $\mathrm{T}$ in addition to the revised cardiac index for peri-operative risk stratification in non-cardiac surgery. Eur Heart J. 2013;34(11):853-62.

Wijeysundera DN. Predicting outcomes: is there utility in risk scores? Can J Anesth. 2016;63(2):148-58.

\section{Publisher's Note}

Springer Nature remains neutral with regard to jurisdictional claims in published maps and institutional affiliations.

Ready to submit your research? Choose BMC and benefit from:

- fast, convenient online submission

- thorough peer review by experienced researchers in your field

- rapid publication on acceptance

- support for research data, including large and complex data types

- gold Open Access which fosters wider collaboration and increased citations

- maximum visibility for your research: over $100 \mathrm{M}$ website views per year

At BMC, research is always in progress.

Learn more biomedcentral.com/submissions 